\title{
Title: Electron-Hole Diffusion Lengths Exceeding 1 Micron in an Organometal Trihalide Perovskite Absorber
}

\author{
Authors: Samuel D. Stranks ${ }^{1}$, Giles E. Eperon ${ }^{1}$, Giulia Grancini ${ }^{2}$, Christopher Menelaou ${ }^{1}$, \\ Marcelo Alcocer ${ }^{2}$, Tomas Leijtens ${ }^{1}$, Laura M. Herz ${ }^{1}$, Annamaria Petrozza ${ }^{2}$, and Henry J. \\ Snaith $^{1 *}$
}

\author{
Affiliations: \\ ${ }^{1}$ University of Oxford, Clarendon Laboratory, Parks Road, Oxford, OX1 3PU, United Kingdom \\ ${ }^{2}$ Center for Nano Science and Technology, @ Polimi, Istituto Italiano di Tecnologia, via \\ Giovanni Pascoli 70/3, 20133, Milan, Italy \\ *Correspondence to: h.snaith1@physics.ox.ac.uk
}

\begin{abstract}
Organic-inorganic perovskites have shown promise as high performance absorbers in solar cells, first as a coating on a mesoporous metal oxide scaffold and more recently as a solid layer in planar heterojunction architectures. Here we report transient absorption and photoluminescence quenching measurements to determine the electron-hole diffusion lengths, diffusion constants, and lifetimes in mixed halide $\left(\mathrm{CH}_{3} \mathrm{NH}_{3} \mathrm{PbI}_{3-\mathrm{x}} \mathrm{Cl}_{\mathrm{x}}\right)$ and triiodide $\left(\mathrm{CH}_{3} \mathrm{NH}_{3} \mathrm{PbI}_{3}\right)$ perovskite absorbers. We find that the diffusion lengths are $>1$ micrometer in the mixed halide perovskite, an order of magnitude greater than the absorption depth. By contrast, the triiodide absorber has electron-hole diffusion lengths of $\sim 100$ nanometers. These results justify the high efficiency of planar heterojunction perovskite solar cells, and identify a critical parameter to optimize for future perovskite absorber development.
\end{abstract}

One Sentence Summary: We use photoluminescence quenching measurements to show that the electron-hole diffusion length in a trihalide perovskite absorber layer exceeds one micron. 


\section{Main Text:}

Photovoltaic (PV) solar energy conversion has the potential to play a major role in future electricity generation. Most currently installed PV arrays consist of crystalline or polycrystalline silicon; in second generation thin-film architectures, light is absorbed and charge generated in a solid layer of this semiconductor (1). Beyond these existing technologies are a myriad of other emerging device concepts based on a broad range of materials, all vying to become the cheapest yet suitably efficient technology to enable widespread uptake of solar energy(2-6). Some of the most promising technologies for ultimate low cost manufacture are solution-processed, such as organic photovoltaics (OPV), dye-sensitized solar cells (DSSCs), and semiconductor-sensitized or extremely thin absorber solar cells $(7,8)$. However, these designs typically require a complex distributed donor-acceptor heterojunction to ionize charge because the exciton and charge carrier diffusion lengths in these materials are much shorter than the absorption depth (7, 9-11). Emerging from the field of DSSCs, the inorganic-organic perovskite family of materials taking the form $\mathrm{ABX}_{3}\left(\mathrm{~A}=\mathrm{CH}_{3} \mathrm{NH}_{3}{ }^{+}, \mathrm{B}=\mathrm{Pb}^{2+}, \mathrm{X}=\mathrm{Cl}^{-}, \mathrm{I}^{-}\right.$, and/or $\left.\mathrm{Br}^{-}\right)$has within the last 12 months been used to fabricate high-performance hybrid solar cells, with reported power conversion efficiencies $(\eta)$ of between 7 and 15\%(12-20). These perovskite absorbers can be solutionprocessed in air and absorb light broadly across the solar spectrum. In the first experiments, the perovskite absorber was used as a sensitizer on mesoporous titania electrodes, along with a solidstate organic hole transporter (HTM) such as 2'-7,7'-tetrakis $(N, N$-di-p-methoxyphenylamine)9,9'-spirobifluorene (spiro-OMeTAD), with the perovskite effectively replacing the dye conventionally employed in a DSSC $(12,13,21)$. Subsequently, the mixed halide $\mathrm{CH}_{3} \mathrm{NH}_{3} \mathrm{PbI}_{3}$ ${ }_{\mathrm{x}} \mathrm{Cl}_{\mathrm{x}}$ perovskite devices have been reported to yield $\eta>12 \%$ when the titania is replaced with an insulating mesoporous alumina scaffold in meso-superstructured solar cells (MSSCs), demonstrating that the perovskite itself can at least sustain sufficient electron transport to enable highly efficient charge collection $(12,16)$. Entirely removing the mesoporous alumina to create a thin film planar heterojunction has resulted in solution processed devices with close to $100 \%$ internal quantum efficiency for charge collection, but with lower $\eta$ of $5 \%$ (16). Very recently, we have demonstrated that if an extremely uniform solid perovskite absorber film can be prepared, for example by vapor deposition, then the highest efficiencies can be achieved in solid thin film planar heterojunction architectures(19).

Despite the rapid increase in efficiency associated with the evolution of this technology, most of the fundamental questions concerning the photophysics and device operation remain unanswered. Arguably the most critical question concerning whether mesostructured or planar heterojunction perovskite solar cells will eventually dominate, is what the exciton or the electron and hole diffusion lengths are in these materials. Here, we perform photoluminescence quenching measurements to extract the electron-hole diffusion lengths in triiodide $\left(\mathrm{CH}_{3} \mathrm{NH}_{3} \mathrm{PbI}_{3}\right)$ and mixed halide $\left(\mathrm{CH}_{3} \mathrm{NH}_{3} \mathrm{PbI}_{3-\mathrm{x}} \mathrm{Cl}_{\mathrm{x}}\right)$ perovskite thin-films. We show that both electron and hole diffusion lengths are $>1 \mu \mathrm{m}$ for the mixed halide perovskite, a factor of $\sim 5$ to 10 greater than the absorption depth. By contrast, the diffusion lengths in the triiodide perovskite are only on the order of or slightly shorter than the absorption depth $(\sim 100 \mathrm{~nm})$. The larger diffusion length in the mixed halide perovskite results from a much longer recombination lifetime and is consistent with far superior performance in MSSCs and planar heterojunction solar cells, as we demonstrate here.

Photoluminescence (PL) quenching has been previously employed successfully with organic semiconductors, in order to determine the diffusion length of the photoexcited bound electron- 
hole pair, the exciton(22). By simply fabricating solid thin films in the presence or absence of an exciton quenching layer, and modelling the photoluminescence decay to a diffusion equation, it is possible to accurately determine the exciton lifetime, diffusion rate and diffusion length(22). However, for the organolead trihalide perovskites studied here, there is relatively little literature on probing the fundamental photophysics, and even the most basic question of whether photoluminescence occurs via free carrier recombination from the conduction and valence band electrons, or is preceded by exciton formation, remains unknown. Exciton binding energies for $\mathrm{CH}_{3} \mathrm{NH}_{3} \mathrm{PbI}_{3}$ have been reported in the range of 37 to $50 \mathrm{meV}$ in the orthorhombic phase (23-25). In principle, at ambient temperature, these values are comparable to thermal energies $\left(\mathrm{k}_{\mathrm{B}} \mathrm{T} \sim 26 \mathrm{meV}\right.$, where $\mathrm{k}_{\mathrm{B}}$ is the Boltzmann constant); thus, both free carriers and weakly bound excitons should co-exist with interchange being possible between the two populations. However, provided all species (bound or free charges) decay with the same rate or through the same channel, the photoluminescence decay should still be representative of recombination or depopulation of electrons and holes from the perovskite film.

The $\mathrm{CH}_{3} \mathrm{NH}_{3} \mathrm{PbI}_{3}$ and $\mathrm{CH}_{3} \mathrm{NH}_{3} \mathrm{PbI}_{3-\mathrm{x}} \mathrm{Cl}_{\mathrm{x}}$ perovskite precursor solutions were spin-coated on plasma-etched glass at room temperature in air, followed by annealing in air at $100^{\circ} \mathrm{C}$ for 45 minutes for the mixed halide and $150^{\circ} \mathrm{C}$ for 15 minutes for the triiodide (these temperatures and times correspond to the optimized conditions for best performance in the solar cells processed in air). Obtaining uniform and continuous perovskite films is essential for the subsequent PL quenching measurements. As we have shown elsewhere, this is possible through precise control of processing conditions (26). In order to obtain air- and moisture-insensitive samples, the neat perovskite films (non-quenching samples) were sealed by spin-coating a layer of the insulating polymer poly(methylmethacrylate) (PMMA) on top. Full experimental details are given in the supplementary materials (SM).

In Figure 1A, we show the UV-Vis absorption and photoluminescence spectra for a $\mathrm{CH}_{3} \mathrm{NH}_{3} \mathrm{PbI}_{3-\mathrm{x}} \mathrm{Cl}_{\mathrm{x}}$ thin film. The photoluminescence is right at the band edge, with very little Stokes-shift. This indicates little vibronic relaxation of the perovskite crystal, unlike organic semiconductors, which typically show large Stokes-shifts(27). Figure 1B shows the ns- transient absorption (TA) spectra of a $\mathrm{CH}_{3} \mathrm{NH}_{3} \mathrm{PbI}_{3-\mathrm{x}} \mathrm{Cl}_{\mathrm{x}}$ film. We observe a sharp negative band peaking at $750 \mathrm{~nm}$ together with a broad positive band at shorter wavelengths, peaking around $550 \mathrm{~nm}$. We assign the negative band to the photobleaching (PB) of the band gap or exciton transition, while the positive band represents a photoinduced absorption (PA). From biexponential data fitting we retrieve the dominant time constant of both the PB and PA to be $\sim 280 \mathrm{~ns}$, followed by a long-lived tail $(>1 \mu \mathrm{s})$. As we show in Figure 1C, the PA band decay (288 $\pm 12 \mathrm{~ns})$ mirrors the PB recovery dynamics $(283 \pm 6 \mathrm{~ns})$, suggesting that the spectra arise from the same population. If we compare the transient absorption decays to the transient photoluminescence decay $\left(\tau_{\mathrm{e}}=273 \pm 7 \mathrm{~ns}\right.$, where $\tau_{\mathrm{e}}$ is the time taken for the PL to fall to 1/e of its initial intensity), there is a strikingly close match. This indicates that the decay of the radiative species we are monitoring with the PL represents the decay of all absorbing species (free carriers or weakly bound excitons) in the perovskite film. We can therefore employ PL quenching measurements to determine a relevant diffusion constant and length in the perovskite films. We cannot however categorically determine whether this corresponds to the diffusion of free charge or excitons, but in either case it will represent the relevant diffusion length for charge extraction in the solar cell. Additionally, we can safely exclude any contribution to the PL from trap states, which would be at lower energy, and whose presence would otherwise complicate the subsequent PL quenching analysis. 


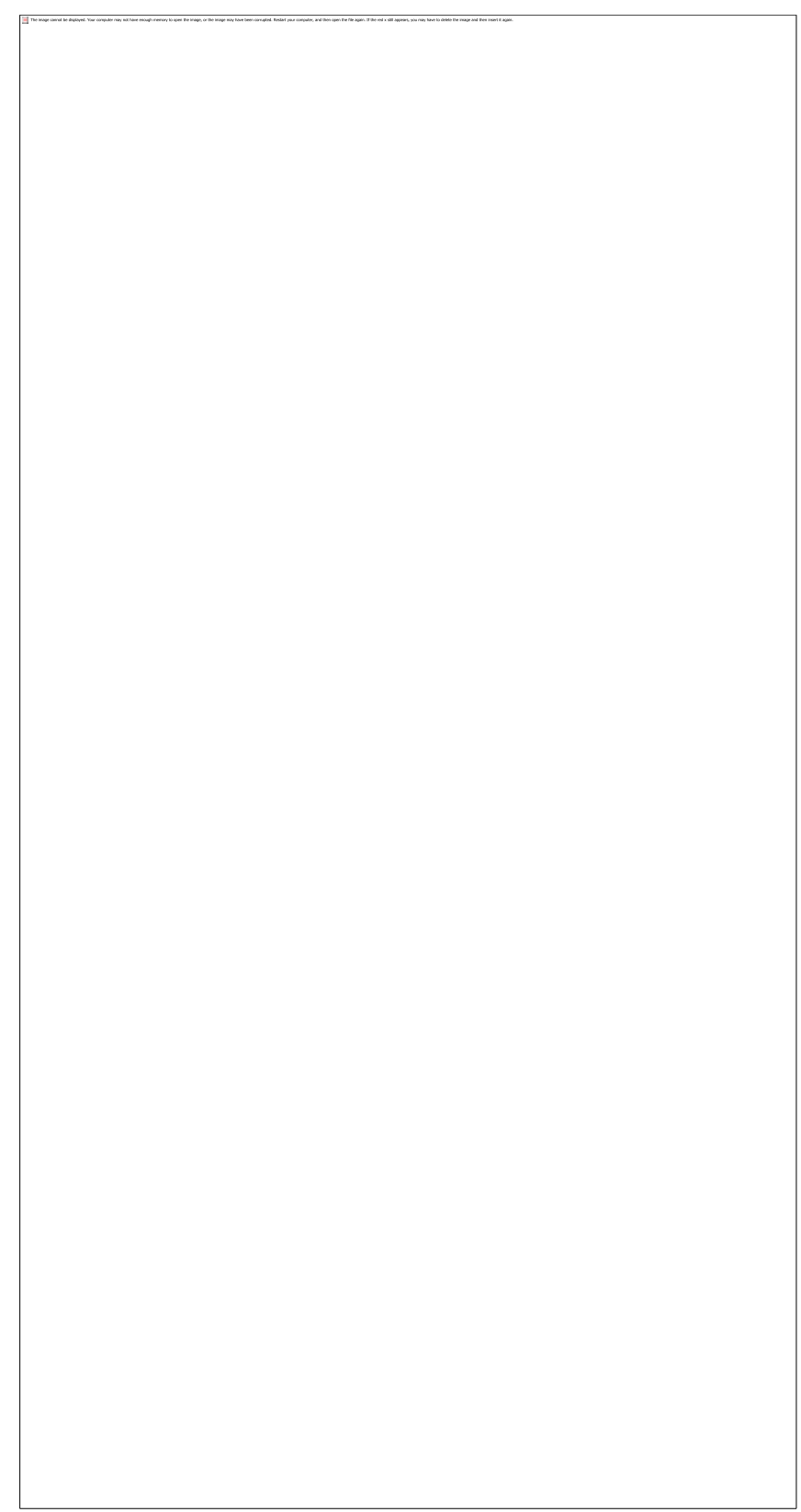

Fig. 1: Optical characterization of the mixed halide perovskite $\mathrm{CH}_{3} \mathrm{NH}_{3} \mathrm{PbI}_{3-\mathrm{x}} \mathrm{Cl}_{\mathrm{x}}$. (A) Absorption (red squares) and photoluminescence (black circles) spectra of a 270-nm thick layer of $\mathrm{CH}_{3} \mathrm{NH}_{3} \mathrm{PbI}_{3-\mathrm{x}} \mathrm{Cl}_{\mathrm{x}}$, coated with PMMA. (B) Transient absorption spectra of $\mathrm{CH}_{3} \mathrm{NH}_{3} \mathrm{PbI}_{3-\mathrm{x}} \mathrm{Cl}_{\mathrm{x}}$ upon excitation at $500 \mathrm{~nm}\left(100 \mu \mathrm{J} / \mathrm{cm}^{2}\right.$ pulses $)$. Each gated spectrum has been integrated for 200 ns, and the PL removed. (C) Normalized photobleaching (PB; red squares, left axis) and PL dynamics (black circles, right axis) probed at $750 \mathrm{~nm}$ and $770 \mathrm{~nm}$, respectively. Biexponential fitting of the PB data (dark red line) leads to a dominant time constant of $\tau_{1}=283 \pm 6 \mathrm{~ns}$, matching the dynamics of the PL $\left(\tau_{\mathrm{e}}=273 \pm 7 \mathrm{~ns}\right)$, followed by a long-lived tail. The inset shows the photoinduced absorption (PA) dynamics at $550 \mathrm{~nm}$ (blue squares) with a biexponential fit (dark blue line; dominant time constant of $\tau_{1}=288 \pm 12 \mathrm{~ns}$ ), also matching the dynamics of the PL and the dominant component of the PB. 
The quenching samples were prepared by spin-coating layers of either a hole-acceptor (SpiroOMeTAD) or an electron-accepting fullerene (phenyl- $\mathrm{C}_{61}$-butyric acid methyl ester; PCBM) on top of the perovskite films. In Figure 2A, we show a scanning electron microscope (SEM) image of a $\sim 270 \mathrm{~nm}$-thick $\mathrm{CH}_{3} \mathrm{NH}_{3} \mathrm{PbI}_{3-\mathrm{x}} \mathrm{Cl}_{\mathrm{x}}$ absorber layer with a $\sim 100 \mathrm{~nm}$ thick Spiro-OMeTAD holequenching layer. We show SEM images of all sample configurations in Figures S1 and S2, and absorption spectra in Figure S3, which indicate that the mixed halide and triiodide perovskites have very similar bandgaps.

We present the time resolved PL decays, measuring the peak emission at $\sim 770 \mathrm{~nm}$, for the mixed halide and triiodide perovskite absorbers in Figures $2 \mathrm{~B}$ and $2 \mathrm{C}$, respectively. We note that the excitation fluence was kept to $0.03 \mu \mathrm{Jcm}^{-2} /$ pulse to ensure that non-linear effects, such as excitoncharge annihilation, are unlikely to occur. The thickness of the $\mathrm{CH}_{3} \mathrm{NH}_{3} \mathrm{PbI}_{3-\mathrm{x}} \mathrm{Cl}_{\mathrm{x}}$ films was $270 \pm 40 \mathrm{~nm}$ and the thickness of the $\mathrm{CH}_{3} \mathrm{NH}_{3} \mathrm{PbI}_{3}$ films was $180 \pm 35 \mathrm{~nm}$, comparable to optimum device thicknesses. The corresponding steady-state spectra are shown in Figure S4. The PL decay of the neat $\mathrm{CH}_{3} \mathrm{NH}_{3} \mathrm{PbI}_{3-\mathrm{x}} \mathrm{Cl}_{\mathrm{x}}$ film exhibits a time-constant of $\tau_{\mathrm{e}}=273 \pm 7 \mathrm{~ns}$. The addition of the PCBM and Spiro-OMeTAD electron and hole quenching layers significantly accelerates the PL decay, with observed time constants $\tau_{\mathrm{e}}$ of $6.1 \pm 0.1 \mathrm{~ns}$ and $5.1 \pm 0.1 \mathrm{~ns}$, respectively. By contrast, the lifetime for the neat $\mathrm{CH}_{3} \mathrm{NH}_{3} \mathrm{PbI}_{3}$ film is only $\tau_{\mathrm{e}}=9.6 \pm 0.3 \mathrm{~ns}$, and this is quenched further but not by such a large fraction, to $3.17 \pm 0.03 \mathrm{~ns}$ for electrons and $4.2 \pm 0.1 \mathrm{~ns}$ for holes. 


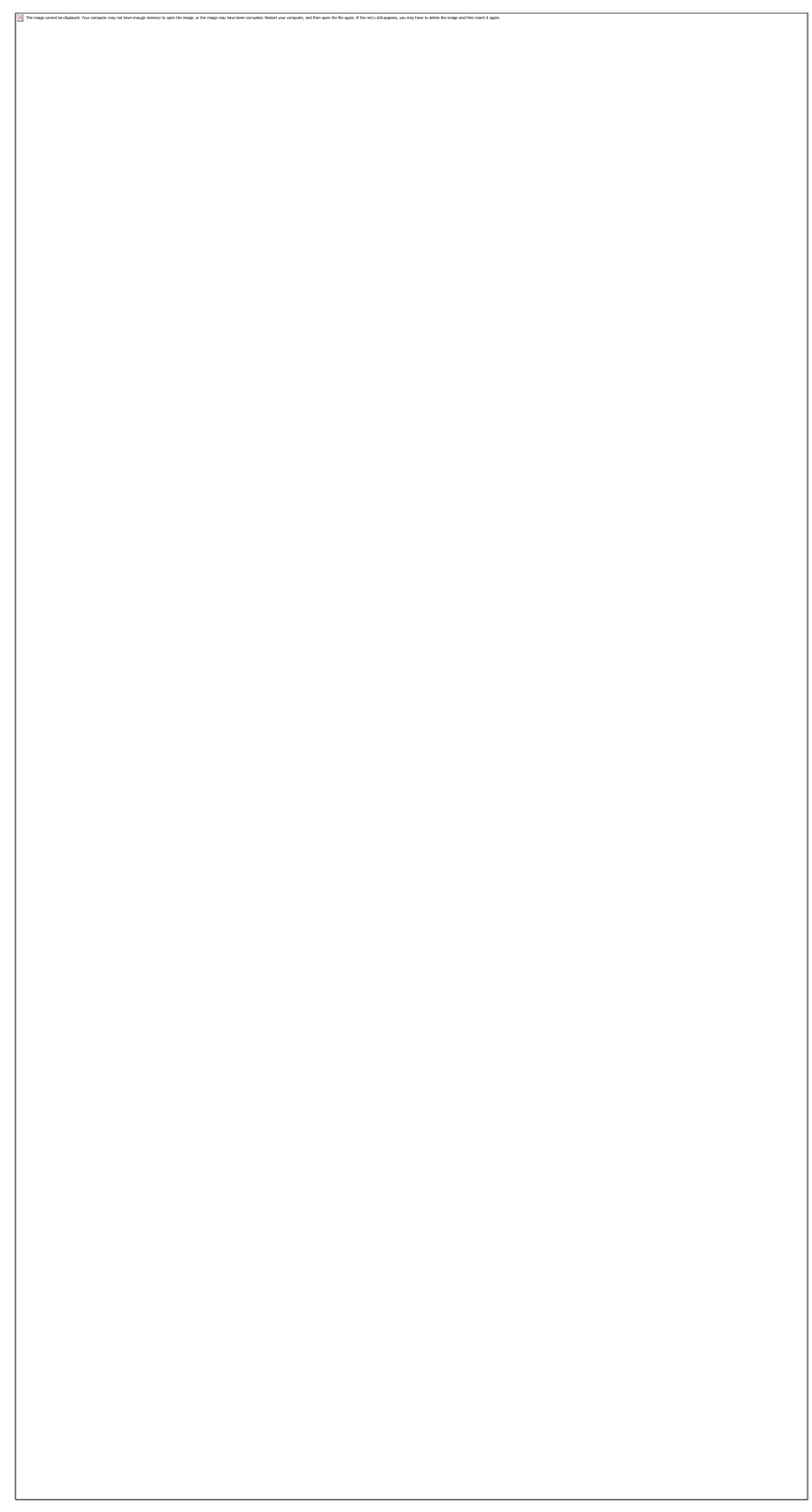

Fig. 2. Photoluminescence measurements and fits to the diffusion model for the mixed halide and triiodide perovskites in the presence of quenchers. (A) Cross-sectional SEM image of a 270-nm thick mixed halide absorber layer with a top hole-quenching layer of Spiro-OMeTAD. (B, C) Time-resolved PL measurements taken at the peak emission wavelength of the (B) mixed halide perovskite and (C) triiodide perovskite with an electron (PCBM; blue triangles) or hole (SpiroOMeTAD; red circles) quencher layer, along with stretched exponential fits to the PMMA data (black squares) and fits to the quenching samples using the diffusion model described in the text (see SM for details). A pulsed (0.3 to $10 \mathrm{MHz}$ ) excitation source at $507 \mathrm{~nm}$ with a fluence of $30 \mathrm{~nJ} / \mathrm{cm}^{2}$ impinged on the glass substrate side. Inset in $(B)$ : Comparison of the PL decay of the two perovskites (with PMMA) on a longer time scale, with lifetimes $\tau_{\mathrm{e}}$ quoted as the time taken to reach $1 / \mathrm{e}$ of the initial intensity. 
The PL decay dynamics were modeled by calculating the number and distribution of excitations in the film $n(x, t)$ according to the 1-D diffusion equation (eq. 1),

$$
\frac{\partial n(x, t)}{\partial t}=D \frac{\partial^{2} n(x, t)}{\partial x^{2}}-k(t) n(x, t)
$$

where $D$ is the diffusion coefficient and $k(t)$ is the PL decay rate in the absence of any quencher material(22) (see SM for further details). The total decay rate $k$ was determined by fitting a stretched exponential decay to the PL data measured from perovskite layers with PMMA. The effect of the quencher-layer was included by assuming that all photogenerated carriers which reach the interface are quenched, giving the boundary condition $n(L, t)=0$, where $x=0$ at the glass/perovskite interface and $L$ is the perovskite film thickness. As the samples were photoexcited from the glass substrate side of the samples, the initial distribution of photoexcitations was given by $n(x, 0)=n_{0} \exp (-\alpha x)$, where $\alpha$ is the absorption coefficient. The average diffusion length $\mathrm{L}_{\mathrm{D}}$ of the species was then determined from $L_{D}=\sqrt{D \tau_{e}}$, where $\tau_{\mathrm{e}}$ is the recombination lifetime in the absence of a quencher. If free charges are predominantly created upon photoexcitation, the PL decay represents the depopulation of charge carriers and we estimate here the diffusion coefficients for holes or electrons depending on which quenching layer is employed. The results from the diffusion model fits are shown in Fig. 2B and C and the parameters summarized in Table 1. Strikingly, the diffusion lengths for both electrons and holes in the mixed halide perovskite are greater than $1 \mu \mathrm{m}$, which is significantly longer than the absorption depth of 100 to $200 \mathrm{~nm}$. This indicates that there should be no requirement for meso or nanostructure with this specific perovskite absorber. By contrast, the triiodide perovskite $\mathrm{CH}_{3} \mathrm{NH}_{3} \mathrm{PbI}_{3}$ films have over one order of magnitude shorter diffusion length of $\sim 100 \mathrm{~nm}$ for both electrons and holes, which is too short with respect to the absorption depth for this material to perform at the highest efficiencies in the thin-film configuration. The close similarity of the derived electron and hole diffusion coefficients and lengths in each of these perovskites either indicates very similar mobility for both electrons and holes, or it indicates that the predominant diffusion species is the weakly bound exciton. We can unfortunately not discriminate between the two from these results.

Table 1. Values for diffusion constants $(D)$ and diffusion lengths $\left(L_{D}\right)$ from fits to PL decays using the diffusion model described in the text. The errors quoted predominantly arise from perovskite film thickness variations, which are $\pm 35 \mathrm{~nm}$ for the triiodide perovskite films and $\pm 40 \mathrm{~nm}$ for the mixed halide perovskite films.

\begin{tabular}{|c|c|c|c|}
\hline Perovskite & Species & $\mathbf{D}\left(\mathbf{c m}^{\mathbf{2}} \mathbf{s}^{\mathbf{- 1}}\right)$ & \multicolumn{1}{c|}{$\mathbf{L}_{\mathbf{D}}(\mathbf{n m})$} \\
\hline \multirow{2}{*}{$\mathrm{CH}_{3} \mathrm{NH}_{3} \mathrm{PbI}_{3-\mathrm{x}} \mathrm{Cl}_{\mathrm{X}}$} & Electrons & $0.042 \pm 0.016$ & $\mathbf{1 0 6 9} \pm \mathbf{2 0 4}$ \\
\cline { 2 - 4 } & Holes & $0.054 \pm 0.022$ & $\mathbf{1 2 1 3} \pm \mathbf{2 4 3}$ \\
\hline \multirow{2}{*}{$\mathrm{CH}_{3} \mathrm{NH}_{3} \mathrm{PbI}_{3}$} & Electrons & $0.017 \pm 0.011$ & $\mathbf{1 2 9} \pm \mathbf{4 1}$ \\
\cline { 2 - 4 } & Holes & $0.011 \pm 0.007$ & $\mathbf{1 0 5} \pm \mathbf{3 2}$ \\
\hline
\end{tabular}

To confirm the importance of the determined diffusion length in full solar cells, and to test whether the measurement correlates well with device results, we fabricated solution-processed thin-film planar heterojunction solar cells with both the mixed halide and triiodide perovskites. We formed solid perovskite films on FTO-coated glass substrates, coated with an n-type $\mathrm{TiO}_{2}$ 
compact layer to ensure selective collection of electrons at the FTO substrate. Subsequently, ptype spiro-OMeTAD was deposited as a hole-transporting layer, to form a planar $\mathrm{p}-\mathrm{i}-\mathrm{n}$ heterojunction architecture. Current-voltage characteristics for the best devices fabricated are shown in Figure 3. The $\mathrm{CH}_{3} \mathrm{NH}_{3} \mathrm{~Pb} \mathrm{I}_{3-\mathrm{x}} \mathrm{Cl}_{\mathrm{x}}$ planar heterojunction solar cells reach power conversion efficiencies in excess of $12 \%$, the highest solution processed planar heterojunction perovskite efficiency reported to date. By contrast, we were only able to attain $\eta$ of $\sim 4 \%$ with the $\mathrm{CH}_{3} \mathrm{NH}_{3} \mathrm{PbI}_{3}$. Upon optimization of devices to obtain the highest efficiencies, we found that a thick layer of $\mathrm{CH}_{3} \mathrm{NH}_{3} \mathrm{~Pb} \mathrm{I}_{3-\mathrm{x}} \mathrm{Cl}_{\mathrm{x}}$ was preferable $(\sim 500 \mathrm{~nm})$. However, the best $\mathrm{CH}_{3} \mathrm{NH}_{3} \mathrm{PbI}_{3}$ cells had a perovskite layer thickness of only $140 \mathrm{~nm}$. This observation is consistent with the diffusion length calculations, where the triiodide films are limited by the $\sim 100 \mathrm{~nm}$ diffusion length, such that photogenerated charge in thicker films cannot be efficiently extracted before recombining. A full set of device results and average performances is given in Table S1.

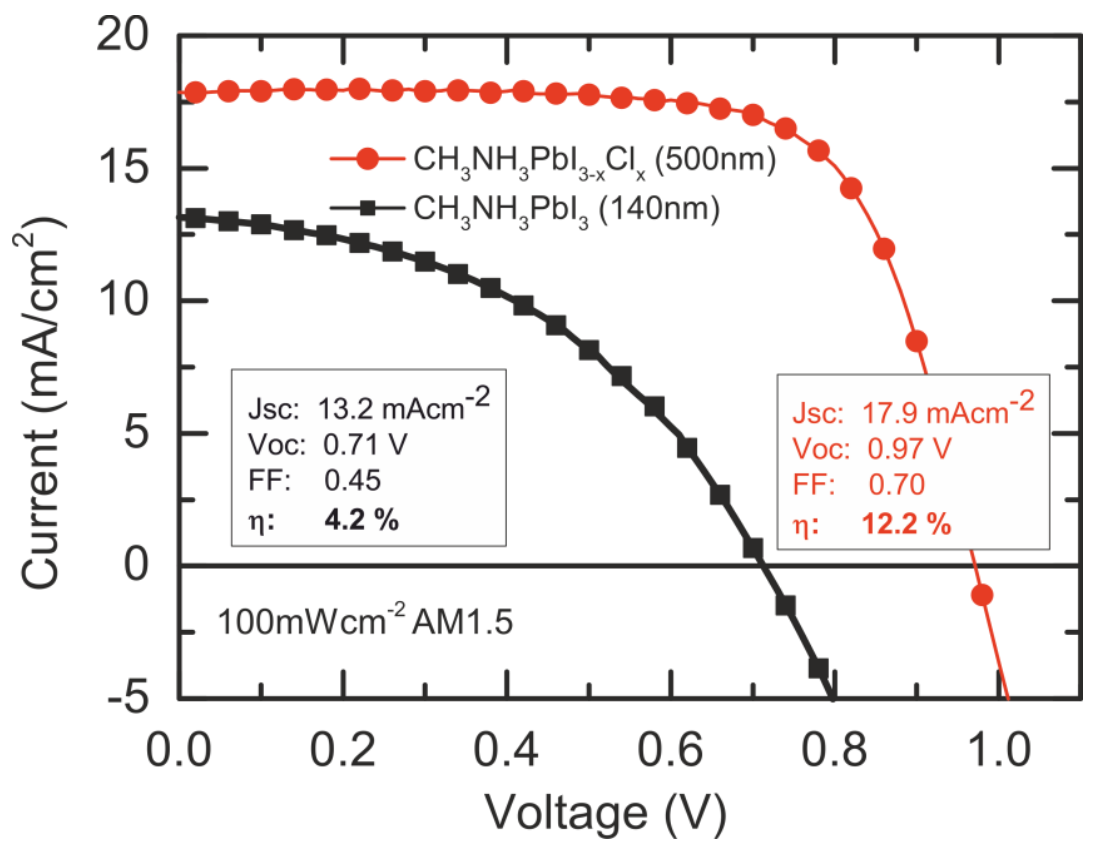

Fig. 3. Current-voltage curves for optimized planar heterojunction perovskite solar cells. $\mathrm{CH}_{3} \mathrm{NH}_{3} \mathrm{PbI}_{3-\mathrm{x}} \mathrm{Cl}_{\mathrm{x}}$ (red line, circle symbols) and $\mathrm{CH}_{3} \mathrm{NH}_{3} \mathrm{PbI}_{3}$ (black line, square symbols) cells were both measured under $100 \mathrm{mWcm}^{-2} \mathrm{AM} 1.5$ simulated sunlight. $\mathrm{J}_{\mathrm{SC}}$ is the short-circuit current, $\mathrm{V}_{\mathrm{OC}}$ is the open-circuit voltage, $\mathrm{FF}$ is the fill factor, and $\eta$ is the power conversion efficiency.

In conclusion, this work indicates that with correct tuning of the perovskite absorber, nano or mesostructure are not necessary in order to achieve highly efficient charge generation and collection. Our results hence pave the way for further advances in planar heterojunction perovskite solar cells. Fundamentally, there still remain many open questions for the community concerning the nature of the excited state, the relative fraction of free and bound charge pairs at room temperature, and the interplay between the two species. Furthermore, this work introduces a new question, as to why the small addition of chloride ions to the organolead triiodide perovskite results in such a striking increase in the electron-hole diffusion length, predominantly arising from a substantial inhibition of non-radiative electron-hole recombination. Understanding these subtleties will enable further improvement of the current family of materials. 


\section{References}

1. S. Chu, A. Majumdar, Opportunities and challenges for a sustainable energy future. Nature 488, 294 (2012).

2. B. O'Regan, M. Gratzel, A low-cost, high-efficiency solar cell based on dye-sensitized colloidal TiO2 films. Nature 353, 737 (1991).

3. Z. He et al., Enhanced power-conversion efficiency in polymer solar cells using an inverted device structure. Nat Photonics 6, 591 (2012).

4. R. A. J. Janssen, J. Nelson, Factors Limiting Device Efficiency in Organic Photovoltaics. Advanced Materials 25, 1847 (2013).

5. T. Todorov, D. B. Mitzi, Direct Liquid Coating of Chalcopyrite Light-Absorbing Layers for Photovoltaic Devices. European Journal of Inorganic Chemistry 2010, 17 (2010).

6. E. H. Sargent, Colloidal quantum dot solar cells. Nat Photonics 6, 133 (2012).

7. M. Graetzel, R. A. J. Janssen, D. B. Mitzi, E. H. Sargent, Materials interface engineering for solution-processed photovoltaics. Nature 488, 304 (2012).

8. G. Hodes, D. Cahen, All-Solid-State, Semiconductor-Sensitized Nanoporous Solar Cells. Accounts of Chemical Research 45, 705 (2012).

9. G. Yu, J. Gao, J. C. Hummelen, F. Wudl, A. J. Heeger, Polymer Photovoltaic Cells: Enhanced Efficiencies via a Network of Internal Donor-Acceptor Heterojunctions. Science 270, 1789 (1995).

10. O. V. Mikhnenko et al., Exciton diffusion length in narrow bandgap polymers. Energy \& Environmental Science 5, 6960 (2012).

11. J. J. M. Halls et al., Efficient photodiodes from interpenetrating polymer networks. Nature 376, 498 (1995).

12. M. M. Lee, J. Teuscher, T. Miyasaka, T. N. Murakami, H. J. Snaith, Efficient Hybrid Solar Cells Based on Meso-Superstructured Organometal Halide Perovskites. Science 338, 643 (2012).

13. H.-S. Kim et al., Lead Iodide Perovskite Sensitized All-Solid-State Submicron Thin Film Mesoscopic Solar Cell with Efficiency Exceeding 9\%. Sci. Rep. 2, (2012).

14. J. H. Heo et al., Efficient inorganic-organic hybrid heterojunction solar cells containing perovskite compound and polymeric hole conductors. Nat Photonics 7, 487 (2013).

15. J. H. Noh, S. H. Im, J. H. Heo, T. N. Mandal, S. I. Seok, Chemical management for colorful, efficient, and stable inorganic-organic hybrid nanostructured solar cells. Nano Letters 13, 1764 (2013).

16. J. M. Ball, M. M. Lee, A. Hey, H. J. Snaith, Low-temperature processed mesosuperstructured to thin-film perovskite solar cells. Energy \& Environmental Science 6, 1739 (2013).

17. J. Burschka et al., Sequential deposition as a route to high-performance perovskitesensitized solar cells. Nature 499, 316 (2013).

18. A. Abrusci et al., High-Performance Perovskite-Polymer Hybrid Solar Cells via Electronic Coupling with Fullerene Monolayers. Nano Letters 13, 3124 (2013).

19. M. Liu, M. B. Johnston, H. J. Snaith, Efficient Planar Heterojunction Perovskite Solar Cells by Vapour Deposition. Nature DOI: 10.1038/nature12509, (2013).

20. W. Zhang et al., Enhancement of Perovskite-Based Solar Cells Employing Core-Shell Metal Nanoparticles. Nano Letters DOI: 10.1021/nl4024287, (2013).

21. A. Kojima, K. Teshima, Y. Shirai, T. Miyasaka, Organometal Halide Perovskites as Visible-Light Sensitizers for Photovoltaic Cells. J. Am. Chem. Soc. 131, 6050 (2009). 
22. P. E. Shaw, A. Ruseckas, I. D. W. Samuel, Exciton Diffusion Measurements in Poly(3hexylthiophene). Advanced Materials 20, 3516 (2008).

23. T. Ishihara, Optical properties of PbI-based perovskite structures. Journal of Luminescence 60-61, 269 (1994, 1994).

24. K. Tanaka et al., Comparative study on the excitons in lead-halide-based perovskite-type crystals CH3NH3PbBr3 CH3NH3PbI3. Solid State Communications 127, 619 (2003).

25. M. Hirasawa, T. Ishihara, T. Goto, K. Uchida, N. Miura, Magnetoabsorption of the lowest exciton in perovskite-type compound (CH3NH3)PbI3. Physica B: Condensed Matter 201, 427 (1994).

26. G. E. Eperon, V. M. Burlakov, P. Docampo, A. Goriely, H. J. Snaith, Morphological Control for High Performance, Solution-Processed Planar Heterojunction Perovskite Solar Cells. Advanced Funtional Materials DOI: 10.1002adfm.201302090, (2013).

27. J. R. Lakowicz, Principles of Fluorescence Spectroscopy. (Springer London, Limited, 2007).

Acknowledgments: This project was funded by the Engineering and Physical Sciences Research Council (EPSRC), the European Research Council (ERC-StG 2011 HYPER Project no. 279881), Oxford Photovoltaics Ltd. through a Nanotechnology KTN CASE award, and by a Royal Society Wolfson exchange grant. The authors thank Valerio D'Innocenzo for technical support and Jack Alexander-Webber for atomic force microscopy supporting measurements. A.P. and H.J.S. thank “The Royal Society International Exchanges Scheme 2012/R2”. Dr. S. Stranks thanks Worcester College, Oxford, for additional financial support.

\section{Supplementary Materials:}

Materials and Methods

Figures S1-S4

Tables S1-S2 